\title{
Piriformis syndrome as the only initial manifestation of septic sacroiliac osteomyelitis
}

\author{
Authors: Mei-Kar Leong ${ }^{A}$ and Poyin Huang ${ }^{B}$
}

\begin{abstract}
Piriformis syndrome refers to sciatica symptoms that do not originate from spinal root compression, but involve the overlying piriformis muscle. Diagnosis is clinical since there are no definitive tests to confirm the diagnosis. Piriformis syndrome is often misinterpreted as sciatica or other pains. Rarely, it could be one of the manifestations of pelvic osteomyelitis. Pelvic osteomyelitis is rare in adults, but it is associated with high morbidity and mortality. One of the severe complications is bacteraemia, which is life-threatening and may be associated with poor outcome. Acute pelvic osteomyelitis is often not initially diagnosed owing to its non-specific symptoms, including fever and severe but poorly localised pain, which may result in a delay of appropriate treatment. Thus, prompt diagnosis is crucial to the prognosis. Here, we report a patient suffering from acute pelvic osteomyelitis with piriformis syndrome as the only initial manifestation without fever. This unusual manifestation rendered prompt and correct diagnosis difficult.
\end{abstract}

KEYWORDS: Piriformis syndrome, magnetic resonance imaging, sciatica, pain, pelvic osteomyelitis

DOI: 10.7861/clinmed.2020-0035

\section{Introduction}

Pelvic osteomyelitis is uncommon, but it is associated with high morbidity and mortality if left untreated. ${ }^{1}$ Life-threatening complications include bacteraemia, which may lead to a poor outcome. The correct diagnosis is usually made ultimately, but it is often initially unrecognised. It can be difficult to diagnose owing to its miscellaneous clinical manifestations, including fever and severe pain. However, the pain may be poorly localised or accompanied by other non-specific symptoms. ${ }^{2}$ In one study, the time interval between initial symptoms and the diagnosis ranged from 1 to 8 days. Difficulty diagnosing may result in delayed treatment. Thus, correct diagnosis of this debilitating disease is

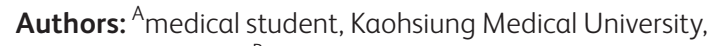
Kaohsiung, Taiwan; ${ }^{B}$ neurologist, Kaohsiung Medical University, Kaohsiung, Taiwan of crucial importance to the prognosis. Here, we report a young immunocompetent patient with no previous underlying disease, suffering from acute septic pelvic osteomyelitis presenting with piriformis syndrome as the only initial manifestation without fever. This unusual manifestation rendered prompt diagnosis of this debilitating disease difficult.

\section{Case presentation}

A 24-year-old man with no underlying disease visited our emergency department due to progressive pain and numbness of his left buttock area with radiation down to his left lower limb for 1 day. History of recent trauma was negative. HIV status and syphilis were checked regularly and the results were all negative. At the emergency department, he was clear with stable vital signs (blood pressure (BP) of $100 / 65 \mathrm{mmHg}$, body temperature (BT) of $36.1^{\circ} \mathrm{C}$, heart rate (HR) of 82 beats per minute and respiration rate (RR) of 19 breaths per minute). Routine blood tests were normal. Movement of his left lower extremity was restricted due to excruciating pain. A lumbar spine $X$-ray was obtained and showed mild L2-L3 and L3-L4 retrolisthesis. An initial diagnosis of sciatica was made and symptomatic treatment was given. He was then discharged from the emergency department. However, due to progressive worsening of his pain (9/9 of visual analogue scale / verbal rating scale), he revisited our hospital the next day. On presentation, he was febrile (BP of $127 / 78 \mathrm{mmHg}, \mathrm{BT}$ of $39^{\circ} \mathrm{C}$, HR of 98 beats per minute and RR of 20 breaths per minute) and had cold sweats. Apart from his pain, physical and neurological examination was otherwise normal. He was thus admitted for further evaluation.

\section{Investigations and diagnosis}

After admission, laboratory investigations revealed markedly elevated C-reactive protein ( $146.2 \mathrm{mg} / \mathrm{L}$ ) without leukocytosis. Blood culture revealed oxacillin-sensitive Staphylococcus aureus. HIV and syphilis status were rechecked, but they remained negative. Hepatitis $B$, hepatitis C, CD4 counts and autoimmune survey revealed non-significant findings. Lumbar spine contrast-magnetic resonance imaging (MRI) revealed left sacroiliitis with osteomyelitis of the left sacrum and ilium (Fig 1). Concurrent myositis over left piriformis, left erector spinae, left iliacus and left iliopsoas muscle were also noted. Thus, the diagnosis of piriformis syndrome secondary to septic sacroiliac osteomyelitis with concurrent piriformis myositis was made. 


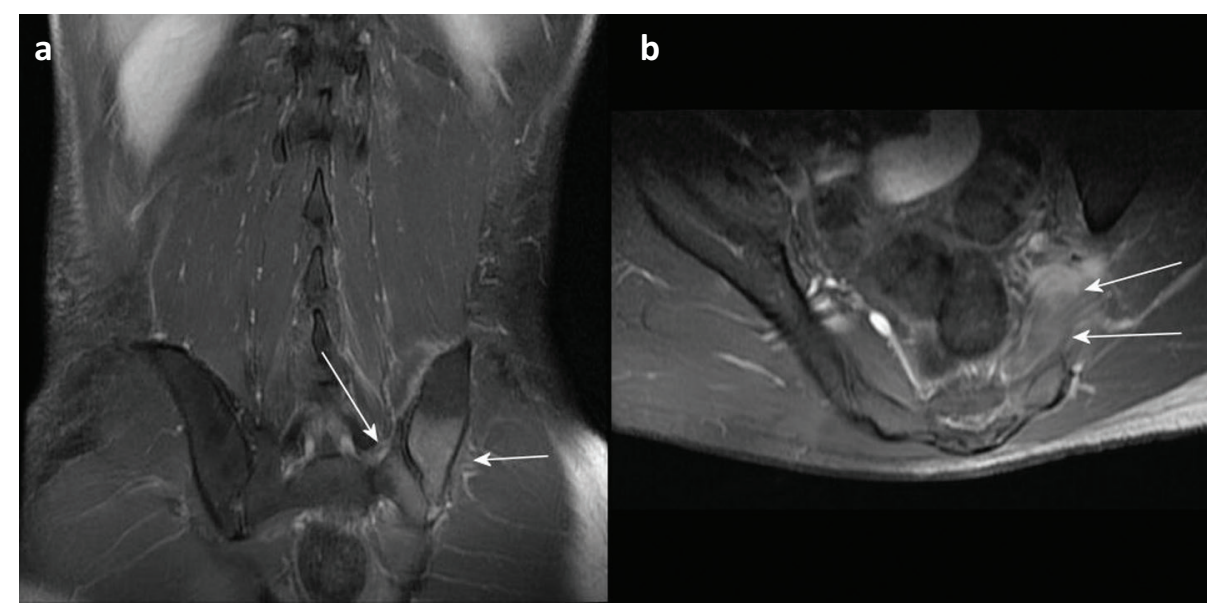

Fig 1. Magnetic resonance imaging of sacrum and ilium. a) Coronal view of presence of bone marrow oedema involving left sacrum and ilium around the sacroiliac joint (arrow) with post-contrast enhancement indicating osteomyelitis. b) Axial view of enhancement of left piriformis muscle (arrow) indicating myositis.

\section{Treatment and outcome}

Oxacillin 2 g every 4 hours was administered for 24 days. The patient's fever subsided and his left buttock and lower limb pain improved significantly after treatment. After being hospitalised for 32 days, he was able to be ambulatory and was discharged. The patient later recovered completely without any known sequelae.

\section{Discussion}

Piriformis syndrome refers to sciatica symptoms that do not originate from spinal root compression, but involve the overlying piriformis muscle. It is caused by compression of the sciatic nerve around the piriformis muscle. ${ }^{3}$ Diagnosis is clinical since there are no specific tests to confirm the diagnosis. Piriformis syndrome is often left undiagnosed, or misinterpreted as sciatica or other pains due to similar symptoms, with pain in the back, quadriceps, lower leg and buttock. ${ }^{4}$ Aetiologies include muscle injury, scoliosis, prolonged sitting, hip surgery, strenuous exercise and piriformis muscle spasm. ${ }^{5}$ Rarely, it can be one of the manifestations of pelvic osteomyelitis with concurrent myositis. ${ }^{2}$ In this case, the patient was initially afebrile and presented to our emergency department with sciatica symptoms. Thus, an initial diagnosis of sciatica was made. However, after the patient revisited the emergency department with unusual excruciating pain and fever, further thorough survey was warranted. Surprisingly, sacroiliac osteomyelitis with concurrent piriformis myositis was later confirmed by MRI in this young immunocompetent patient. The treatment of pelvic osteomyelitis is challenging owing to the anatomic constraints of the pelvis and the high degree of comorbidity in affected patients. ${ }^{1}$ Thus, early diagnosis and appropriate treatment with the adequate antibiotic course are essential to reduce morbidity and mortality. Pelvic osteomyelitis is Mei-Kar Leong and Poyin Huang in adults, and our patient only presented initially with sciatica symptoms without fever. ${ }^{1}$ This unusual manifestation of uncommon disease rendered prompt diagnosis difficult.

\section{Learning points}

> Pelvic osteomyelitis can occur in young immunocompetent individuals without identifiable aetiologies.

$>$ When encountering patients presenting with sciatica symptoms without fever, alterations of clinical symptoms and signs should be carefully followed, especially when the pain is excruciating.

> In patients presenting with sciatica symptoms and fever, MRI plays a crucial role in diagnosis.

\section{References}

1 Dudareva M, Ferguson J, Riley $N$ et al. Osteomyelitis of the pelvic bones: a multidisciplinary approach to treatment. J Bone Jt Infect 2017:2:184-93.

2 Phadke PS, Gandhi AR, More SA, Joshirao RP. Salmonella pyomyositis with concurrent sacroiliac osteomyelitis presenting as piriformis syndrome: a rare case. J Postgrad Med 2017;63:44-6.

3 Cass SP. Piriformis syndrome: a cause of nondiscogenic sciatica. Curr Sports Med Rep 2015;14:41-4.

4 Probst D, Stout A, Hunt D. Piriformis syndrome: a narrative review of the anatomy, diagnosis, and treatment. PM R 2019;11(Suppl 1): S54-63.

5 Fishman LM, Hosseini M. Piriformis syndrome - a diagnosis comes into its own. Muscle Nerve 2019;59:395-6.

Address for correspondence: Dr Poyin Huang, Kaohsiung Municipal Siaogang Hospital, 482 Shanming Road, Siaogang District, Kaohsiung City 812, Taiwan.

Email:u99802003@gmail.com 